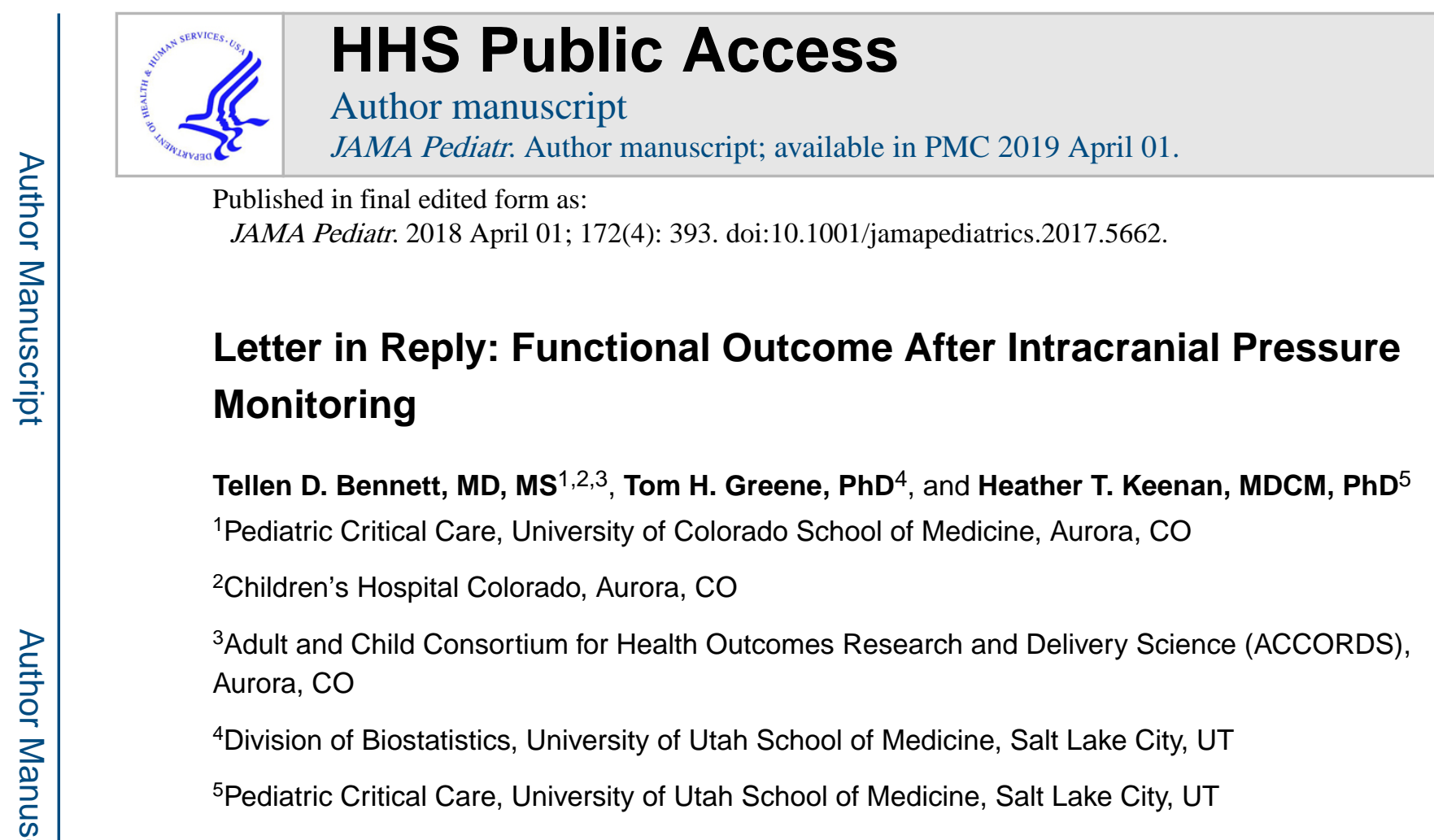

\title{
Keywords
}

pediatrics; craniocerebral trauma; brain injuries; intracranial hypertension

\section{Letter in Reply}

We appreciate the letter by Figueiredo et al. discussing our recent manuscript. ${ }^{1}$ The letter's authors raised three important points.

First, they asked questions about our decision to analyze the 118 patients who received intracranial pressure (ICP) monitors $\geq 24$ hours after admission with the no-monitor group. Observational studies attempting to make causal inferences when the treatment is administered at different times must consider the potential for "immortal time" bias. ${ }^{2,3}$ In our study, an ICP monitor could not be received $\geq 24$ hours after admission unless the patient had survived until the monitor was placed. If we had counted those receiving the ICP monitor $\geq 24$ hours after admission among the treated subjects, we would have created an immortal time bias in favor of the treatment, as some treated subjects would have had "guaranteed survival" to the time of placement. To address this bias, we used a recommended analytic approach ${ }^{2}$ that mimics a randomized trial. We defined a "treatment period," during which subjects are placed into treatment groups according to the treatments they received during that period, and a subsequent "observation period" during which subjects are observed for the occurrence of outcomes. Analogous to intent-to-treat analyses in randomized trials, our treatment effect estimates may have been attenuated slightly by the 118 "cross-overs" of initially untreated patients who later received ICP monitors after 24 hours. However, because these 118 patients represent just $5.7 \%$ of our 2,085 untreated

Corresponding Author: Tellen D. Bennett, MD, MS, Pediatric Critical Care, University of Colorado School of Medicine, Children's Hospital Colorado, Adult and Child Consortium for Health Outcomes Research and Delivery Science (ACCORDS), 13199 E. Montview Blvd, Suite 300, Campus Mail F443, Aurora, CO 80045, tell.bennett@ucdenver.edu. 
subjects, the cross-over rate was too small to have appreciably affected our results. The external validity of the manuscript's main results was strengthened, not compromised as the authors suggested, by this analytic approach.

Second, the authors correctly pointed out that the two treatment groups had important differences in both injury characteristics and subsequent therapeutic intensity (eTable 4 and Table 2). They commented that more children in the ICP monitor-treated group received inotropes or vasopressors than those in the no-monitor group. We agree that, taken together, differences in treatment intensity suggest that despite the seemingly excellent covariate balance we obtained, the propensity weighting approach did not completely equate the two treatment groups. We explored that idea in the manuscript's Discussion and the authors of the manuscript's accompanying editorial ${ }^{4}$ also discussed it.

Finally, Figueiredo et al. called for a randomized trial of ICP monitoring in children with TBI. We have held preliminary discussions about such a trial with leading TBI investigators. The consensus thus far has been that, despite our work ${ }^{1}$ and others' ${ }^{5}$, there is insufficient equipoise in North America. If experts such as Figueiredo et al. feel that the necessary equipoise exists in South America, we would happily collaborate with them to design and conduct a trial. Indeed, a team of North and South American investigators recently conducted a trial of ICP monitoring in adults with TBI in Bolivia and Ecuador. ${ }^{6}$ We respect the international multidisciplinary TBI research community's commitment to answering the field's most challenging questions and improving the outcomes of children and adults who suffer TBI.

\section{Acknowledgments}

Funding: This work was supported by the Eunice Kennedy Shriver National Institute for Child Health and Human Development (Grant K23HD074620 to TB).

\section{References}

1. Bennett TD, DeWitt PE, Greene TH, et al. Functional outcome after intracranial pressure monitoring for children with severe traumatic brain injury. JAMA pediatrics. 2017; 171(10):965-971. DOI: 10.1001/jamapediatrics.2017.2127 [PubMed: 28846763]

2. Zhou Z, Rahme E, Abrahamowicz M, Pilote L. Survival bias associated with time-to-treatment initiation in drug effectiveness evaluation: A comparison of methods. Am J Epidemiol. 2005; 162(10):1016-1023. http://www.ncbi.nlm.nih.gov/entrez/query.fcgi? $\mathrm{cmd}=$ Retrieve $\& \mathrm{db}=$ PubMed\&dopt=Citation\&list_uids=16192344. [PubMed: 16192344]

3. Suissa S. Immortal time bias in pharmaco-epidemiology. Am J Epidemiol. 2008; 167(4):492-499. http://www.ncbi.nlm.nih.gov/entrez/query.fcgi? $\mathrm{cmd}=$ Retrieve $\& \mathrm{db}=$ PubMed\&dopt=Citation\&list_uids=18056625. [PubMed: 18056625]

4. Horvat CM, Kochanek PM. Big data not yet big enough to determine the influence of intracranial pressure monitoring on outcome in children with severe traumatic brain injury. JAMA pediatrics. 2017; 171(10):942-943. DOI: 10.1001/jamapediatrics.2017.2390 [PubMed: 28846750]

5. Alali AS, Gomez D, Sathya C, et al. Intracranial pressure monitoring among children with severe traumatic brain injury. J Neurosurg Pediatr. 2015; 16(5):523-532. DOI: 10.3171/2015.3.PEDS14507

6. Chesnut RM, Temkin N, Carney N, et al. A trial of intracranial-pressure monitoring in traumatic brain injury. N Engl J Med. 2012; 367(26):2471-2481. DOI: 10.1056/NEJMoa1207363 [PubMed: 23234472] 